\title{
Virtuelle Medien in den Ingenieurwissenschaften: Lernen mit neuen Medien
}

\author{
Petra Maier
}

Das Internet und digitale Medien erhalten zunehmend eine zentrale Funktion bei der gesellschaftlichen Kommunikation von Wissen und sind zu einem Träger von Bildung und Kultur geworden. Auch Hochschulen setzen verstärkt diese Komponenten ein, um Lerninhalte mehrdimensional aufbereitet in Höchstgeschwindigkeit übertragen zu können und den Studenten zur Verfuigung zu stellen. Die neuen Medien eröffnen ungeahnte Möglichkeiten für das heute unabdingbare lebenslange Lernen. Wissen soll spielerisch, einfach, intuitiv, aber auch effizient, kostenoptimierend und in Zeiten der Globalisierung unabhängig von Zeit, Ort und Datenmengen erworben und genutzt werden.

Der Prozess von „Wissen überall und zu jeder Zeit“ muss aktiv gestaltet werden, dessen Herausforderungen angenommen und die Konsequenzen erkannt werden. Im Moment wird an jeder Lehreinrichtung uiber das „ob überhaupt“ und wenn ja mit welchen Gestaltungsmöglichkeiten und in welchem Rahmen diskutiert. Über die Attraktivität des Lernens zu jeder Zeit und an jedem Ort sollten sich allerdings alle einig sein. Daraus ergeben sich nun zahlreiche Fragen: Wie verändern sich die Kommunikationsprozesse zwischen Lehrenden und Lernenden? Wie beeinflussen die Möglichkeiten der Digitalisierung die Lehrinhalte? Die Frage bezieht sich auf einen möglichen Wissenswandel durch den eintretenden Medienwandel. Welche Medienkombination bietet Lernenden wie Lehrenden die beste Unterstuitzung? Mittelpunkt aller Diskussionen ist heutzutage, wie überzeugend die Visionen fuir die neuen Lernumgebungen sind. Es ist also zu klären, ob es sinnvoll ist, den „Tafel- und Kreide-Professor" in einem kurzen oder langen Prozess über den „PowerPoint-Professor“ in den kompletten „MultimediaProfessor" umzuwandeln.

Es wird oft die Meinung vertreten, dass derjenige, der nichts zu sagen hat, in seiner Vorlesung eine PowerPointPräsentation benutzt. Das ist natürlich eine sehr grobe Beschuldigung derjenigen, die die Qualität ihrer Vorlesung auch durch den Einsatz neuerer Medien versuchen zu verbessern. Aber gehören nicht wenigstens ein paar Abbildungen in die Präsentation, die ohne PowerPoint nur schwierig darstellbar wären?

Oft wird auch auf die Wichtigkeit der Abschreibezeit hingewiesen. Von „der Hand in den Kopf “-Lehrmethode ist dann die Rede, die Lernenden brauchen Zeit zur Verinnerlichung des Lehrstoffes, er soll nicht nur an ihnen vorbeirauschen und dann erst wieder in der Prüfungsphase auftauchen und wirken wie „nie vorher gesehen“. Die Bereitstellung des Lehrstoffes an die Studenten ist vielfältig. Oft sind die Studenten zum Mitschreiben animiert, oft legen die Lehrenden auf reines Zuhören Wert und teilen zusätzlich Scripte aus, oder die Unterlagen werden auf einem Server hinterlegt.

Wesentlich für alle Überlegungen zum Einsatz Neuer Medien im Unterricht sind die Akzeptanz bei Lehrenden und Lernenden, also die Frage nach dem Mehrwert gegenuiber herkömmlichen Methoden und Materialien, sowie die praktische Möglichkeit der Nutzung. Selbstverständlich soll kein Lehrender zur Nutzung von neuen Medien gezwungen werden, auch mit Tafel und Kreide lässt sich didaktisch wertvoller Unterricht machen. Es ist auch bekannt, dass die Vorbereitung von Lehrveranstaltungen aufwendiger wird, wenn man von Tafel und Kreide abweicht. Aber sind wir den jungen Studenten nicht ein bisschen „Neues“ schuldig?

Berechtigt ist auch die Angst vor leeren Vorlesungsräumen, wenn man die Lehrinhalte vorzeitig zur Verfuigung stellt. Der Lehrende sollte immer noch der ,guide by the side" sein, sonst wird die Wissensaufnahme ziellos. Man hört auch oft von Studenten, dass es ihnen schwer falle, in der Prüfungsvorbereitungsphase für mehr als zwei Prüfungen „on screen“ zu lernen. Sie wünschen sich oft noch die Druckvariante, die allerdings dem ,papierlosen“ Unterrichtsraum widerspricht.

Bis zum vollausgerüsteten Vorlesungsraum mit z. B. „Tablet PC“ ist es noch weit. Ob es größerer Anstrengungen bedarf, die Barrieren in den Köpfen, die eine Sinnigkeit dessen in Frage stellen, abzubauen oder die finanziellen Mittel aufzubringen, wird sich bald zeigen. Es sollte nicht vergessen werden, dass wir es mit jungen, modernen und wissbegierigen Studenten zu tun haben, die im High-Tech-Zeitalter aufwachsen. Für diese Generation ist Multimedia Gang und Gebe, so wie sie Software mit Leichtigkeit über „Try and Error“ erlernen.

\section{Umsetzung an der TFH Wildau}

Auch an der Technischen Fachhochschule in Wildau findet ein Medienwandel statt. Alle multimedialen Angebote gewährleisten, dass das Wissen nicht auf der Strecke bleibt.

Ein Beispiel dafür ist der „Basic Support for Cooperative Work“ (BSCW) Server. Dieses System unterstuitzt die Zusammenarbeit durch die Bereitstellung eines gemeinsamen Arbeitsraumes im Internet. Ein geteilter Arbeitsbereich erlaubt Ablage und Aufruf von Dokumenten und 
gemeinsamer Bearbeitung innerhalb einer Gruppe. Diese Funktionalität wird in einen Ereignismechanismus integriert, um jeden Benutzer mit einem Bewusstsein der Tätigkeiten von anderen innerhalb des Arbeitsbereichs zu versehen. Der Server enthält zahlreiche Eigenschaften: z. B. Unterstuitzung für verzweigte Diskussionen, Management der Dokumente, Suchfunktionen und vieles mehr. Das System wurde hauptsächlich entwickelt, um selbst organisierende Gruppen zu stiitzen. Die BSCWEntwicklungen sind teilweise durch die Europäische Union (das CoopWWW-Projekt und das CESAR-Projekt des Telematik-Anwendungsprogramms der EU) finanziert worden. Partner dieser Projekte trugen zur Entwicklung des Systems bei.

BSCW unterstuitzt sowohl die asynchrone und als auch die synchrone Zusammenarbeit mit Partnern über das Internet, im Intranet oder in einem Netz mit Kollegen (,Extranet“).

Für die asynchrone (nicht-gleichzeitige) Zusammenarbeit bietet BSCW gemeinsame Arbeitsbereiche, in denen Gruppen ihre Dokumente ablegen, verwalten, gemeinsam bearbeiten und austauschen können. Die wesentlichen Vorteile des BSCW-Arbeitsverzeichnisses sind, dass Arbeitsgruppen Dokumente gemeinsam nutzen, unabhängig von dem Computersystem, mit dem die Mitglieder arbeiten. Die Mitglieder müssen dafür keine Software installieren, sie brauchen nur einen der marktführenden, frei erhältlichen Web-Browser. Es kann auf BSCWArbeitsbereiche zugegriffen, Ordner-Übersichten können durchgesehen und Dokumente auf den eigenen Rechner heruntergeladen werden wie von „normalen“ Seiten im WWW. Der BSCW informiert uiber alle wichtigen Ereignisse in gemeinsamen Arbeitsbereichen. Dokumente können mit einem Web-Browser in einem gemeinsamen Arbeitsbereich ablegt werden.

Für die synchrone (gleichzeitige) Zusammenarbeit bietet BSCW Werkzeuge für die Planung und Organisation von Treffen, den technischen Aufbau 'virtueller' Treffen auf der Basis von Conferencing-Programmen oder per Telefon, ad hoc-Kommunikation mit Partnern, die gerade in einem gemeinsamen Arbeitsbereich aktiv sind, also im weitesten Sinne an der gemeinsamen Aufgabe arbeiten.

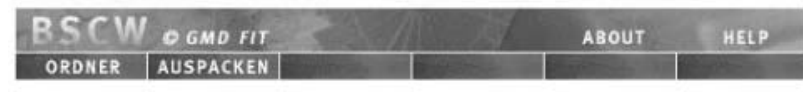

\section{(i) is :username}

Sortieren: [nach TVD] [nach Bewertung] [nach Name] [nach Datum]

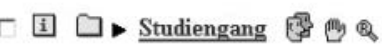

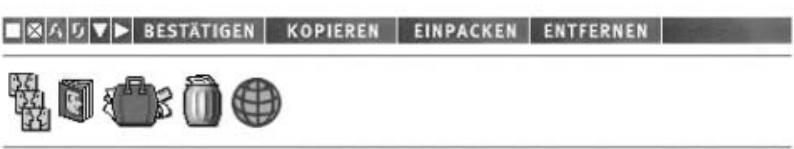

Sie sind: 目

BSCW $\odot$ 2095-2000 GMD

Abb. 1: Übersicht der BSCW-Server-Benutzeroberfläche (www.bscw.de)
Abbildung 1 zeigt die Benutzeroberfläche des BSCWServers an der Technischen Fachhochschule Wildau. Der Kopfbereich einer Ordnerübersichtsseite wird in der Kopfzeile mit dem Benutzernamen angezeigt [hier username]. Die obere Menüleiste zeigt Menüs zum Handhaben von Objekten in Ordnern. In der darauffolgenden Navigationsleiste findet man den Verzeichnispfad bzw. die gegenwärtige Position [hier Studiengang]. Die Menüs werden in der unteren Menüleiste nochmals wiederholt. Ordner und Objekte können nach verschiedenen Sortierkriterien sortiert angezeigt werden. Aktionsflächen (siehe Symbole dem Studiengang folgend) erlauben im aktuellen Ordner [hier Studiengang] das Erzeugen von Objekten wie Dokumenten, Ordnern oder Diskussionen, den Start einer Suche oder den Zugriff auf Informationen über den aktuellen Ordner. Die Fußzeile ist die Schnellzugriffsleiste, und es wird ein Zugriff auf persönliche Objekte wie Kalender, Hauptordner, Papierkorb und Ablage gewährt.

In vielen Fachbereichen wird der BSCW-Server an der Technischen Fachhochschule Wildau erfolgreich eingesetzt. Dozenten stellen den Studenten ihre Unterrichtsmaterialien zur Verfuigung und die einzelnen Studiengänge hinterlegen Informationen über den Studienablauf, Veranstaltungshinweise, Praktikumangebote und Zusammenfassungen von Präsentationen in der Öffentlichkeit.

Weiterhin hat die „e-learning“-Plattform CLIX Campus der Firma IMC AG in die Lehre und Administration der Technischen Fachhochschule Wildau Einzug erhalten (denkbar als Virtuelle Hochschule, als Fernstudienanbieter, als Ergänzung zum Präsenzstudium oder im Rahmen von hochschuluibergreifenden Verbundprojekten). CLIX Campus bietet eine Plattform zur Initiierung, Steuerung und Administration von Lehr- und Lernprozessen und wird bereits im Fernstudium und Präsenzstudium erfolgreich genutzt.

Der Einsatz der Software „FAVORIT ${ }^{\circledR}$-OfficeFlow ${ }^{\circledR}$ “ soll in diesem Beitrag nur kurz genannt werden, um zu unterstreichen, dass multimediale Komponenten den Unterricht sehr lebendig gestalten können und damit die Motivation der Lernenden erhöht wird. Favorit ist das flexible Archivierungs- und Vorgabebearbeitungssystem im IT-gestuitzten Geschäftsgang, welches im Fachbereich „Wirtschaft, Verwaltung und Recht“ angewendet wird. Die Studierenden können in verschiedene Rollen schlïpfen, um Schritte im Verwaltungsablauf über das ,papierarme Büro“ zu simulieren. In ersten Simulationsobjekten wurden Teile der Hochschulverwaltung simuliert. FAVORIT $^{\circledR}$-OfficeFlow ${ }^{\circledR}$ ist Testsieger nach den Kriterien des Konzeptes „Papierarmes Büro“ des Bundesministerium des Innern.

Durch den Einsatz der FAVORIT ${ }^{\circledR}$-OfficeFlow ${ }^{\circledR}$-Software wird auch die soziale Komponente gefördert: Lernende sind aufgefordert miteinander zu arbeiten, sich zu verständigen und in virtuellen Gruppen zu agieren. Dies ist als großer Vorteil von multimedialen Plattformen bekannt. [TFH -INFO Wildau 4/2002] 


\section{Virtuelle Medien im Projekt}

\section{Projektinhalt}

Das Projekt „Virtuelle Medien in den Ingenieurwissenschaften" an der Technischen Fachhochschule Wildau wird im Rahmen des Hochschulsonderprogramms des Landes Brandenburg „Chancengleichheit für Frauen“ durchgefuihrt und von Frau Prof. Dr. Asta Richter geleitet. Das Projekt begann im Juli 2001 und endet im Dezember 2003. Der Hauptinhalt dieses Projektes ist das Erstellen von multimedialen e-learning-Plattformen. Den Studenten soll die Möglichkeit gegeben werden, den Vorlesungsstoff elektronisch aufgearbeitet jederzeit und an jedem Ort nutzen zu können. Auch sollen die Bedingungen fuir die Nutzung beachtet werden; das heißt, die Studenten sollen auch lokal (ohne Verbindung zum Internet) in der Lage sein, auf die Daten zuzugreifen. Weiterhin sind multimediale Komponenten in die Vorlesungen und den Laborbetrieb einzubauen. Ein PC-Arbeitsplatz mit attraktiver Multimedia-Software steht dem Projekt zur Verfuigung. Weiterhin kann auf eine digitale Kamera zurückgegriffen werden, um kleine Filme einzubinden. Diese können geschickt mit den theoretischen Lehrinhalten verknüpft werden.

Einer der größten Vorteile von multimedialen Lernkomponenten ist die Interaktivität, das heißt die Möglichkeit von Feedback, wodurch das Leistungsvolumen der Lernenden erhöht wird. „Learning by doing“ hat an Guiltigkeit nicht verloren. Wird man nun wirklich schlau durch den Mausklick? Der Einbau von Fragen in Tutorials erweist sich als großer Vorteil. Dem Studenten kann sich erst seine eigenen Gedanken machen und dann nach der Antwort suchen. Es hat sich auch als lehrreich herauskristallisiert, die einfachen „Weiter“-Befehle durch eine Auswahl von Themen zu ersetzen. Der Lernende muss nun über den Ablauf seines Tutorials selbst nachdenken.

Viele Firmen und Institute beschäftigen sich momentan mit der Erstellung von e-learning-Plattformen. Neben dem individuellen Lernen wird auch das kooperative Lernen durch Chats, Instant Messaging und Foren unterstuitzt. Unterstuitzung finden die Lernenden durch elektronische und menschliche Tutoren. Im Rahmen des Projektes Virtuelle Medien an der Fachhochschule Wildau entschied man sich für die elektronische Plattform des individuellen Lernens und den Dozenten als „menschlichen“ Tutor. Die erstellten Plattformen bieten dem Studenten ein attraktives Tutorial zum individuellen Lernen in starker Anlehnung an die Vorlesungsinhalte.

\section{Lehrinhalte in „Multimedia“}

Das erste hier erwähnte Tutorial ist das zur Vorlesung „Virtuelle Realität“. Die Vorlesung wird im Studiengang Telematik im vier Semester gehalten. Die Lehrinhalte reichen von der technischen Entwicklung über Anwendungsfelder bis hin zur virtuellen Gesellschaft. Abbildung 2 zeigt das Frontcover zur CD mit der Darstellung eines virtuellen Büros. Nun könnte der Lernende mit seinem Laptop auch von zu Hause aus der Vorlesung fol-

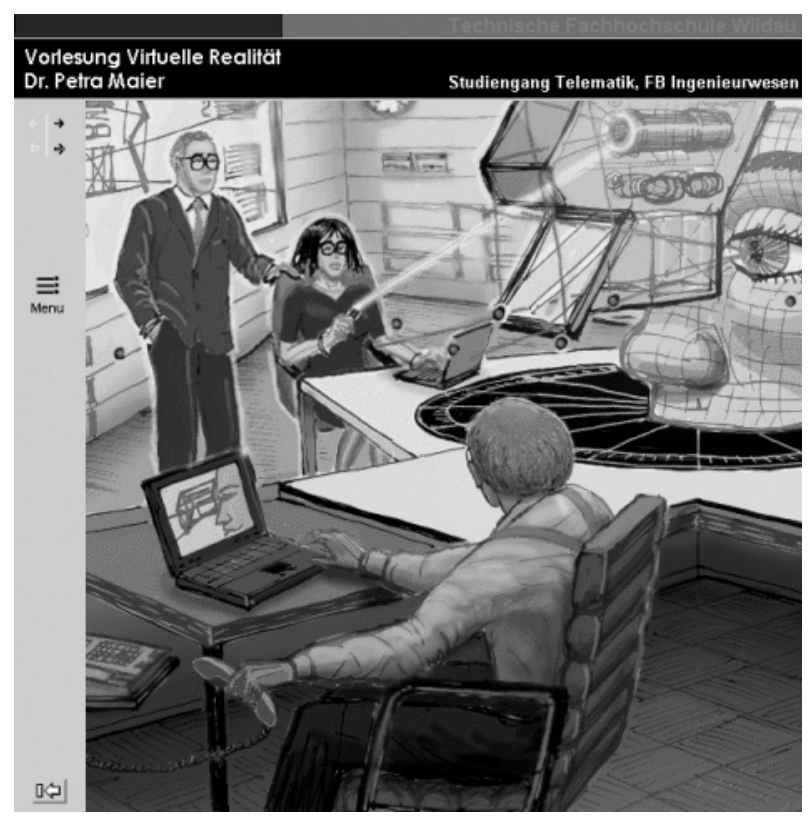

Abb. 2: Frontcover des Tutorials „Virtuelle Realität“ zum elektronischen Lernen im Studiengang Telematik

gen. Multimedia zeigt also deutlich eine Auflösung der Trennung von Räumen, ob nun Arbeitsraum, Vorlesungssaal oder zu Hause. Dieses Tutorial wurde mit Hilfe der Software Macromedia Authorware erstellt.

Abbildungen 3 und 4 zeigen Ausschnitte aus dem Tutorial „Virtuelle Realität“. Dem Lernenden kann sich weitere Informationen zu einer Grundseite anzeigen zu lassen. Die Knöpfe sind deutlich als Aktionsfelder zu erkennen, da sich der Cursor der Maus in eine Hand umwandelt. Nutzt der Student dieses Tutorial nun als Prüfungsvorbereitung, kann er sich zum angegebenen Stichwort erst selbst Gedanken machen und dann die Definition anzeigen lassen. Im Beispiel der Abbildung 3 geht es um die Faktoren, die die virtuelle Einbindung bestimmen. Die Auswahl fiel auf das Blickfeld [field of view], siehe dazu die Markierung. Es erscheint die Erläuterung in der unteren Bildhälfte neben den Auswahlknöpfen: Wieviel Blickfeld wird durch ein Bild gefuillt? An dem Beispiel wird auch deutlich, dass dieses Tutorial in deutscher und englischer Sprache erstellt ist. Abbildung 4 zeigt eine weitere Seite aus dem Tutorial „Virtuelle Realität“. Hier wird die virtuelle Medizin (der virtuelle Patient) behandelt. Über neun verschiedene Auswahlknöpfe lassen sich

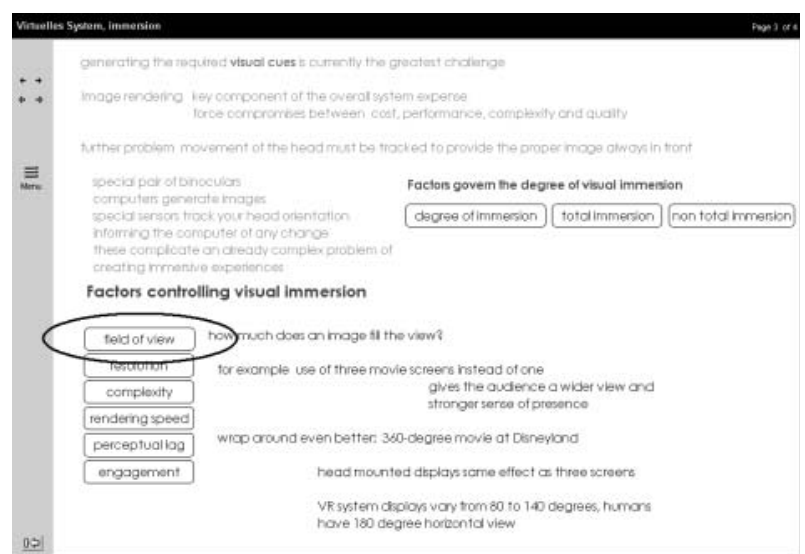

Abb. 3: Auszug aus dem Tutorial „Virtuelle Realität“ zur Immersion im virtuellen System: Darstellung der Auswahlmöglichkeiten; Studiengang Telematik 


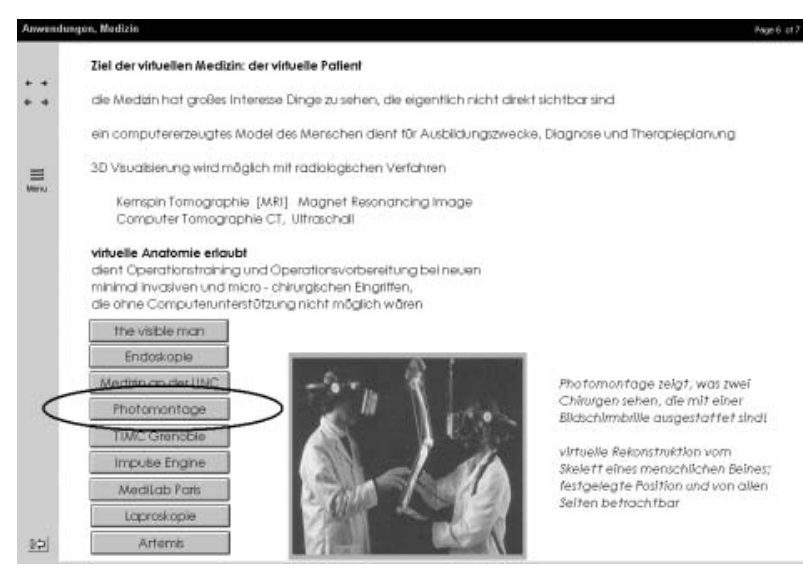

Abb. 4: Auszug aus dem Tutorial „Virtuelle Ralität“ zur virtuellen Medizin: Darstellung der Auswahlmöglichkeiten; Sutdiengang Telematik

weitere Informationen anzeigen. Der Einführungsteil zum Thema bleibt auf der Seite der Übersicht halber stehen. So ist immer eine Zuordnung zum Thema gegeben. Auch hier besteht die Möglichkeit, sich Informationen erst nach Selbstuiberlegung anzeigen zu lassen. Es gibt keine eigentliche „Weiter“-Taste. Die linke feststehende Spalte fuihrt durch die Oberthemen und Unterthemen hin und zurück. Mit Hilfe des Menüpunktes kann die Reihenfolge selbst gewählt werden und ein Springen im Tutorial nach Eigeninteresse wird ermöglicht.

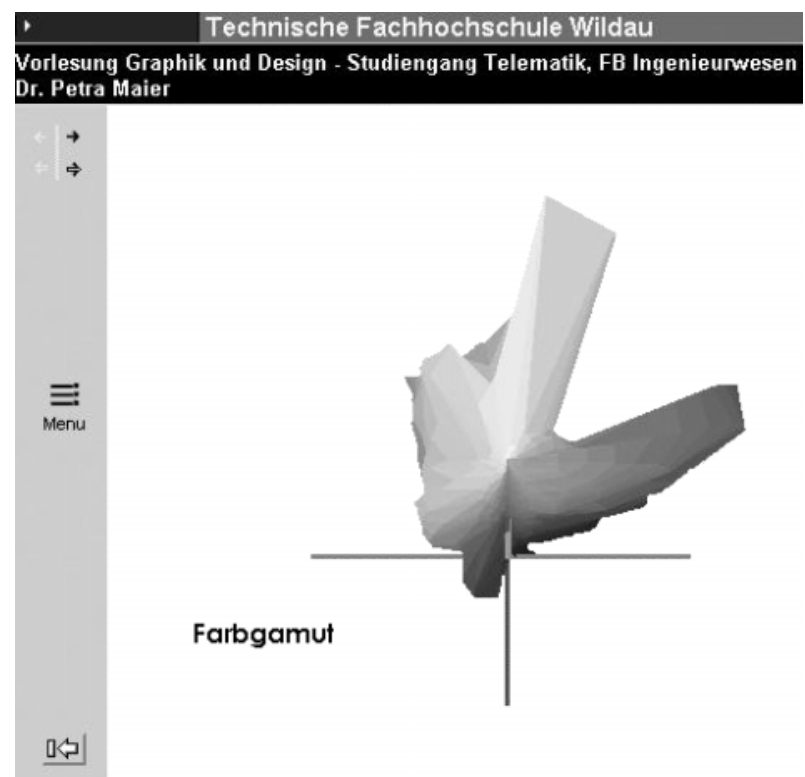

Abb. 5: Frontcover des Tutorials „Graphik und Design“ zum elektronischen Lernen im Studiengang Telematik

Abbildung 5 zeigt das Frontcover zur CD „Graphik und Design“ mit der Darstellung eines Farbgamuts. Dieses Lehrfach wird im Rahmen des Projektes im Studiengang Telematik (5. Semester) unterrichtet. Die Farbenlehre sowie Typographie und Layout sind Schwerpunkte und auch eine wichtige Voraussetzung zur Erstellung von multimedialen Lernkomponenten. Das Grundgerüst des Tutorials ist an alle anderen Tutorials angepasst, um eine Einheitlichkeit zu gewährleisten.

Ein Kernstuick der Tutorials ist das Menui, es bietet eine bedienerfreundliche Navigation durch die Inhalte. Die Ober- und Unterthemen sind im Menü nicht nur aufgelistet, sondern auch direkt anwählbar. Abbildung 6 zeigt das Menüi zum Tutorial „Graphik und Design“. Unterthemen sind versetzt zum Oberthema gezeigt, so zum Beispiel die Unterthemen Titel, Primärfarben, Sekundärfarben usw. zum Oberthema Farbenlehre. Die ausgewählte Seite erscheint sofort im aktuellen Frame. In der Kopfzeile sind die jeweiligen Themen und deren Umfang eingeblendet (hier Unterthemaseite 16 von 26 Seiten).

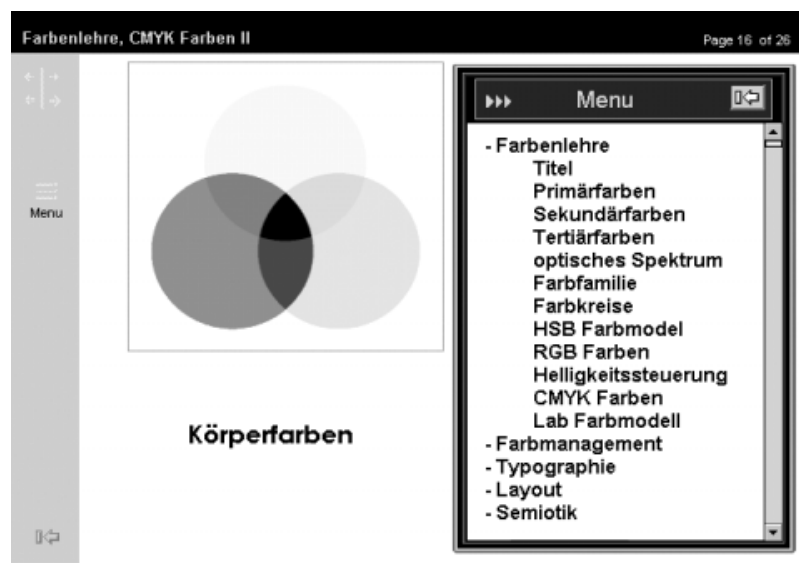

Abb. 6: Tutorial „Graphik und Design“ zu Druckfarben CMYK in der Farbenlehre: Darstellung des Menüs zur Navigation; Studien-gang Telematik

Bereits erwähnt wurde die Wichtigkeit des Einbeziehens von Fragen. Abbildung 7 zeigt einen weiteren Auszug aus dem Tutorial „Graphik und Design“. Die Lernenden können sich erst selbst Antworten überlegen und dann die Auflösung anzeigen lassen. Weiterhin werden komplexe Sachverhalte an einfachen und alltäglichen Gegebenheiten erläutert, so zum Beispiel die Frage nach dem Aussehen der Zitrone. Dass diese Frucht gelb ist, ist allgemein bekannt. Dass die Zitronenschale Lichtanteile unterschiedlich absorbiert und reflektiert, ist auch noch jedem klar. Dass das Absorbieren des blauen Lichtanteils mit der subtraktiven Farbmischung gleichzusetzen ist und so auch die normalen Druckfarben (Körperfarben) arbeiten, ist meist etwas Neues. Ebenso lässt sich die additive Farbmischung am Beispiel Zitrone einfach erklären. Die Lernenden verinnerlichen die Mischung aus Rot und Grün zur Farbe Gelb durch ,educational games“ und verstehen dadurch die Farbmischung von RGB-Farben (Lichtfarben) an z. B. Monitoren besser.

„Gaming for learning“ ist pädagogisch immer noch sehr wertvoll. Erinnern wir uns an die Software FAVORIT ${ }^{\circledR}$.

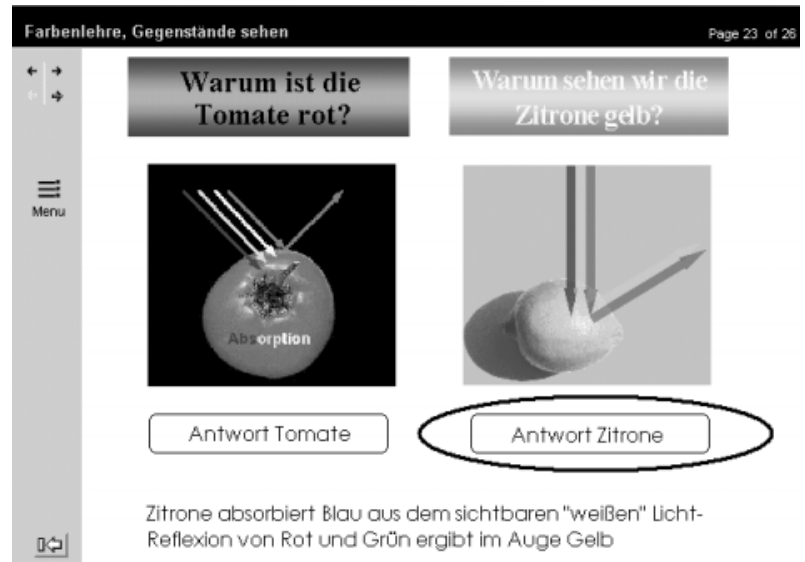

Abb. 7: Tutorial „Graphik und Design“ zur Farbenlehre: eingebaute Fragen; Studiengang Telematik 
OfficeFlow ${ }^{\circledR}$. Lernende sind aufgefordert in virtuellen Gruppen einerseits die Hochschulverwaltung, andererseits die Software selbst zu verstehen. Das Rollenspiel sollte nach der 13 . Klasse nicht aufhören. Semestervorträge lassen sich gut als Jobinterviewvorträge verkaufen und das Arbeiten in Gruppen bei semesterbegleitenden Projekten und Belegen erhöht auf jeden Fall die Sozialkompetenz des einzelnen Studierenden.

Zwei weitere Tutorials zum Thema „Dünnschichttechnik“ und „Nanotechnologie“ fuir den Studiengang Physikalische Technik sind in Arbeit. Für weitere Tutorials sind in Absprache mit den Fachdozenten Konzepte zu erarbeiten.

\section{Vorlesungs- oder laborbegleitende multimediale Möglichkeiten}

Die folgenden multimedialen elektronischen Lernkomponenten wurden mit Hilfe verschiedener SoftwareProgramme erstellt. Macromedia Flash und Adobe Premiere fanden hauptsächlich ihren Einsatz für die vorlesungs- oder laborbegleitenden Lernkomponenten. Diese dienen nicht unbedingt dem elektronischen Selbststudium, sondern der Dozent nutzt diese Multimediakomponente, um die Vorlesung oder das Laborpraktikum zu bereichern. Durch den Einbau von Filmen lassen sich Maschinen oder Apparaturen nicht nur als Einzelbild zeigen, sondern die Wirkungsweise kann auch in Wort und Bild erklärt werden. Ob der Dozent zum Film referiert oder dieser Film durch ihn oder andere dokumentiert wird, ist der Situation anzupassen.

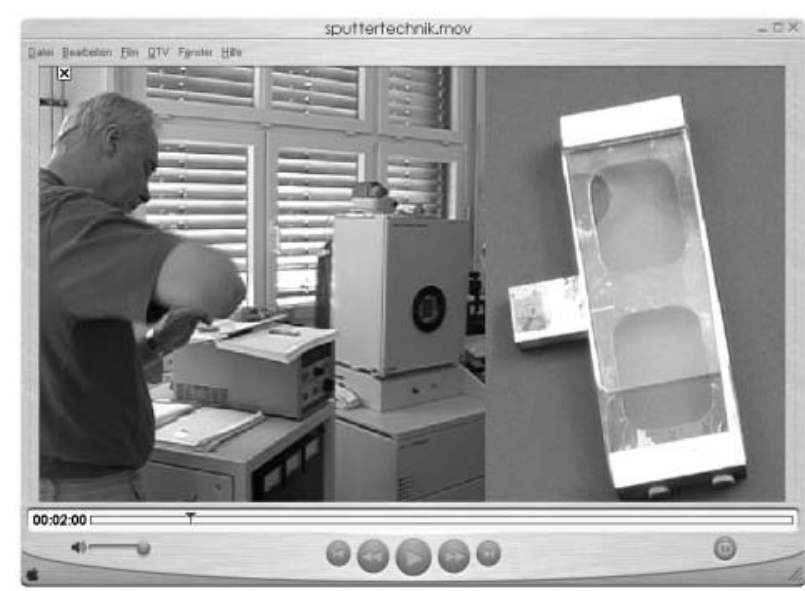

Abb. 8: Auszug aus der multimedialen Lernkomponente zum Laborpraktikum zur Sputtertechnik im Labor für Oberflächentechnik; Studiengang Physikalische Technik

Die ersten dieser vorlesungs- oder laborbegleitenden Lernkomponenten zum Praktikum in der Physikalischen Technik sind fertig gestellt. Abbildung 8 zeigt einen Ausschnitt aus der Lernkomponente zum Laborversuch „Sputtertechnik“. Der linke Teil des Bildes stellt einen Ausschnitt aus einem digital aufgezeichneten Laborversuch dar, welcher in dem Moment von einem Standbild uiberblendet wird. Die Überblendung geschieht meistens mit einem animierten Übergang. Die Lernkomponente „Sputtertechnik“ besteht somit aus Filmen und Standbildern, die sinnvoll miteinander verknüpft sind. Die Filme sind einschließlich dem Ton übernommen, dieser muss allerdings nicht genutzt werden.
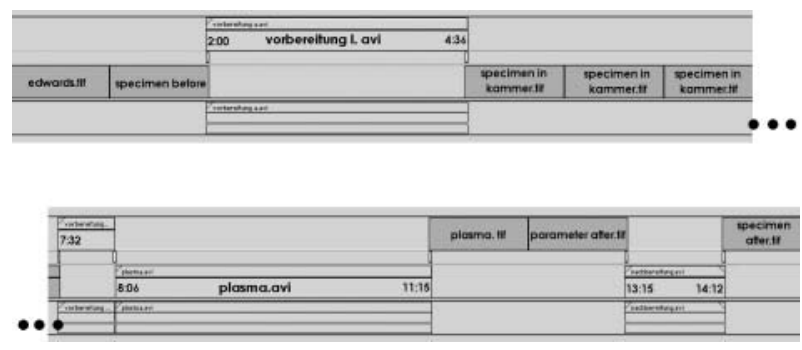

Abb. 9: Übersicht der multimedialen Lernkomponente zum Laborpraktikum zur Sputtertechnik im Labor für Oberflächentechnik des Studigangs Physikalische Technik

Die Zeitlinie der Lernkomponente ist in Abbildung 9 dargestellt. Mit Hilfe dieser Zeitlinie können sich Dozent und Lernende einen Überblick über die Anordnung und deren Zeitrahmen verschaffen. Die Reihenfolge von Einzelbildern und Filmen ist schnell erfassbar. Der Einbezug von Filmen ist an konkreten Zeitfenstern zu erkennen. Für Einzelbilder steht jeweils ca. eine Minute zur Verfuigung. Diese Zeit soll dem Vortragenden zur Erläuterung des Gezeigten zur Verfuigung stehen. Ist dieser Zeitraum zu kurz, kann der Film auch jederzeit gestoppt werden. Für den Einsatz im Selbststudium (in der Annahme, der Student konnte an dem besagten Laborpraktikum nicht teilnehmen) lassen sich Einzelbilder auch vorspulen. Die Einzelkomponenten sind jeweils mit Namen genannt, um eine Art Inhaltsverzeichnis zur Verfuigung zu stellen.

Diese multimediale Lernkomponente kann nicht nur im Laborpraktikum, sondern auch in Vorlesungen eingesetzt werden. Sie lässt sich auch gut auf die Vorbereitung und Einfuihrung in Experimente im Grundlagenpraktikum der Physikalischen Technik oder anderer Studiengänge anwenden. Somit können Experimente und Darstellungen praxisnah in den Vorlesungsraum gebracht werden, ohne dass sich eine Heerschar von Studenten zu den technischen Anwendungen begibt. Im Rahmen eines Laborpraktikums ist dies natürlich gewollt, aber im Rahmen einer Firmenbesichtigung nicht unbedingt immer organisatorisch möglich und oft auch nicht gewünscht. Man sollte die Tatsache nicht außer acht lassen, dass Exkursionen den Studenten ermöglichen, Apparaturen und Verfahren live in voller Größe und Beschaffenheit zu sehen. Dies fördert die Vorstellungskraft und ist ein wichtiger Aspekt im Verstehen von technischen Abläufen. So wird im Fach „Virtuelle Realität“ im Studiengang Telematik jedes Jahr eine Exkursion durchgefuihrt, um sich Geräte und Labore näher anzuschauen. Auch ein Besuch im Labor zur Medizintechnik steht auf dem Programm. Sollte uns diese interessante Möglichkeit nicht mehr zur Verfügung stehen, könnte man den „Besuch“ auch multimedial in die Vorlesung einbringen.

Im letzten Beispiel soll eine kurze Animation beschrieben werden, die als Teil einer Einfuihrungsvorlesung zum Thema „Nanohärte“ im Labor für Oberflächentechnik im Studiengang Physikalische Technik genutzt wird. Die multimediale Komponente wurde mit der Software Macromedia Flash erstellt und als Movie abgespeichert (siehe Abbildung 10). Bekannt sind solche „Intros“ von animierten Internetseiten. In einem kurzen Film werden das verwendete Gerät und erläuternde Folien zu den 


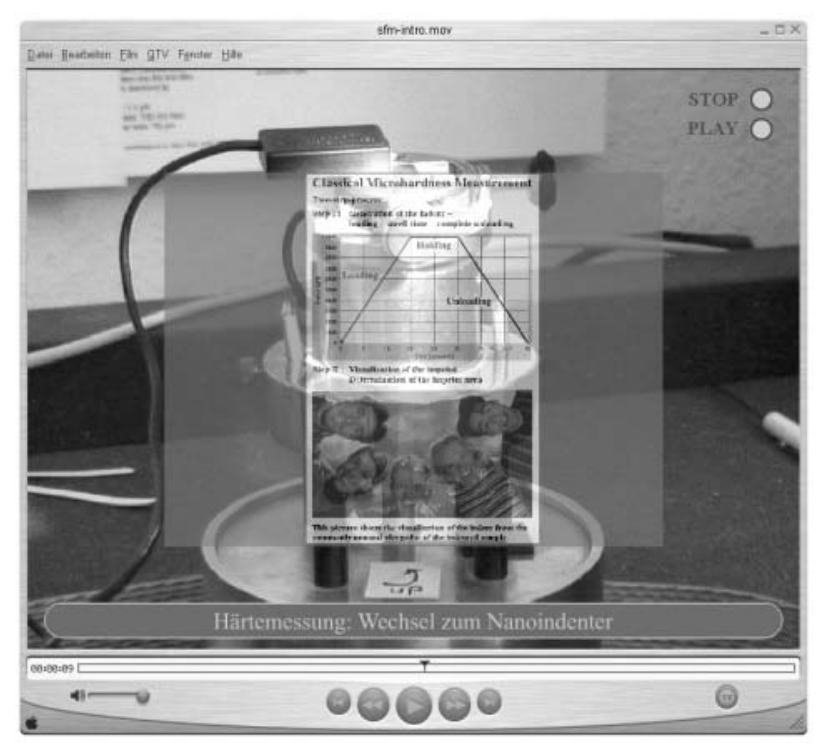

Abb. 10: Multimediale Lernkomponente (Intro) zum Laborpraktikum zur Nanohärte im Labor für Oberflächentechnik; Studiengang Physikalische Technik

Grundlagen der Nanohärtemessung gezeigt. Es gibt die Optionen Stop und Play über die gesamte Länge des Filmes. In dieser Form wurde auch ein Intro zur Sputtertechnik erarbeitet (siehe Abbildung 11).

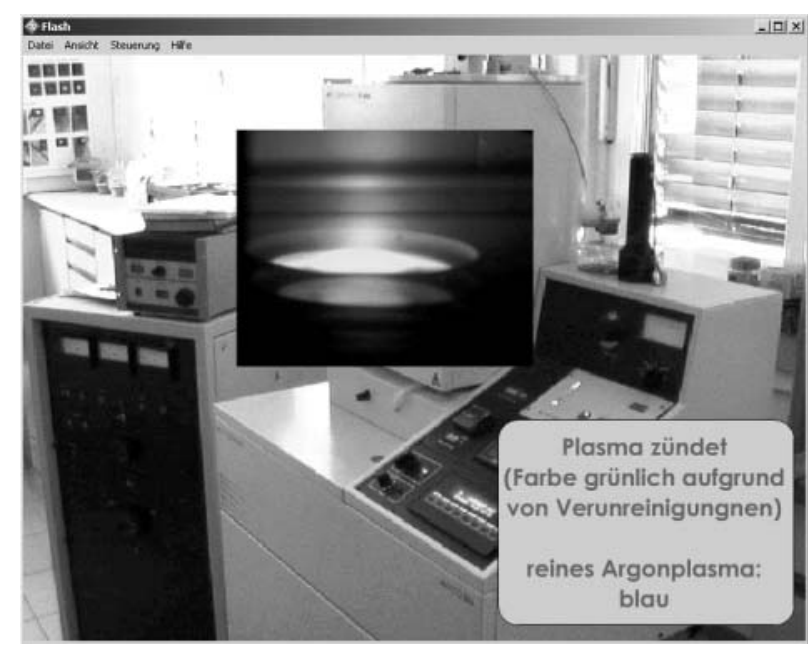

Abb. 11: Multimediale Lernkomponente (Intro) zum Laborpraktikum zur Sputtertechnik im Labor für Oberflächentechnik; Studiengang Physikalische Technik

Das Projekt virtuelle Medien in den Ingenieurwissenschaften befindet sich nun im letzten Drittel der Projektzeit. Weitere Projekte sollen sich nicht nur auf die Studienrichtung „Physikalische Technik“ und den Studiengang „Telematik“ beziehen, sondern auch auf weitere Themen aus den Ingenieurwissenschaften.

\section{Zusammenfassung}

Es konnte gezeigt werden, dass multimediale Lernkomponenten an der Technischen Fachhochschule Wildau nicht nur im Rahmen des Projektes „Virtuelle Medien“ eine wichtige Rolle spielen. Der BSCW-Server bietet eine ausgezeichnete Plattform zur Zusammenarbeit durch die Bereitstellung von gemeinsamen Arbeitsräumen über das Internet. Vorlesungsskripte (vom Dozenten auf dem Server hinterlegt) können vom Studenten überall abgerufen werden. Das Projekt „Virtuelle Medien“ gibt den neuen
Medien durch die Bereitstellung von e-learning-Komponenten zum Selbststudium eine andere attraktive Richtung. Die Aufarbeitung der Tutorials mit Hilfe von Interaktionen und Fragestellungen erweist sich als pädagogisch wertvoll. Weiterhin können Vorlesungen durch den Einsatz von Filmkomponenten und Flashanimationen bereichert werden. Der Einsatz von Multimedia-Komponenten sollte allerdings gut durchdacht werden und eine gewisse Notwendigkeit mit sich bringen.

Eine Umfrage an der Technischen Fachhochschule Wildau hat ergeben, das viele Dozenten bereits Komponenten wie den BSCW-Server, Videosequenzen, Flash-Filme, PowerPoint-Präsentationen und Simulationsprogramme einsetzen, um ihre Vorlesungen zu bereichern. Der pädagogische Aspekt der Wissensvermittlung darf dabei allerdings nicht verloren gehen. Laborexperimente und Exkursionen in Multimedia-Form haben Eintritt in den Vorlesungssaal und schließlich in die Vorlesungsskripte der Studenten erhalten. Weiterhin wird im Fernstudium die e-learning-Plattform Clix Campus der Firma IMC AG zur Gestaltung von „Online-Universitäten“ für die virtuelle Lehre eingesetzt. Diese Plattform soll auch auf das Direktstudium übertragen werden. Erste Versuche wurden erfolgreich durchgeführt. Dem neuen Trend von „Anywhere and Anytime“-Learning steht also auch in Wildau nichts mehr im Wege!

\section{Quellen}

Labor für Oberflächentechnik der TFH Wildau (Abb. 8, 10, 11) Petra Maier: persönl. Notizen vom „Education Quality Forum zu Neuen Medien in der Bildung“, Dortmund Nov. 2002

Margit Scholl/Sabine Schröter: Medienintegrierende und praxisorientierte Verwaltungsinformatik, in: TFH-INFO Wildau $4 / 2002$

Bodo Wolf: Folien eines Vortrags auf der Frühjahrstagung des Arbeitskreises „Rastersondenmikroskopie“, BAM Berlin Febr. 2002 (Abb. 10)

www.bscw.de (Abb. 1)

www.im-c.de (e-learning-Plattform Clix Campus)

\section{Danksagung}

Die hier dargestellten Lernkomponenten konnten erstellt werden durch die Finanzierung und Förderung im Rahmen des Hochschulsonderprogramms des Landes Brandenburg „Chancengleichheit für Frauen“, Frauenförderung HSP-N Projekt Nr. 7. Besonderen Dank an die Projektleiterin Prof. Dr. Asta Richter und das „Telematik“-Team fuir interessante Diskussionen und die gute Zusammenarbeit.

\section{Autor}

\section{Dr. Petra Maier}

Technische Fachhochschule Wildau

Fachbereich Ingenieurwesen/Wirtschaftsingenieurwesen Telefon +493375 508-359

E-Mail: pmaier@igw.tfh-wildau.de 